\title{
Patterns of undertreatment among patients with acute myeloid leukemia (AML): considerations for patients eligible for non-intensive chemotherapy (NIC)
}

\author{
Elizabeth Hubscher ${ }^{1}$ (D) Slaven Sikirica ${ }^{2} \cdot$ Timothy Bell $^{2} \cdot$ Andrew Brown $^{2} \cdot$ Verna Welch $^{2} \cdot$ Alexander Russell-Smith $^{2}$. \\ Paul D'Amico ${ }^{2}$
}

Received: 3 May 2021 / Accepted: 4 August 2021 / Published online: 30 August 2021

(c) The Author(s) 2021

\begin{abstract}
Acute myeloid leukemia (AML) is a life-threatening malignancy that is more prevalent in the elderly. Because the patient population is heterogenous and advanced in age, choosing the optimal therapy can be challenging. There is strong evidence supporting antileukemic therapy, including standard intensive induction chemotherapy (IC) and non-intensive chemotherapy (NIC), for older patients with AML, and guidelines recommend treatment selection based on a patient's individual and disease characteristics as opposed to age alone. Nonetheless, historic evidence indicates that a high proportion of patients who may be candidates for NIC receive no active antileukemic treatment (NAAT), instead receiving only best supportive care (BSC). We conducted a focused literature review to assess current real-world patterns of undertreatment in AML. From a total of 25 identified studies reporting the proportion of patients with AML receiving NAAT, the proportion of patients treated with NAAT varied widely, ranging from 10 to $61.4 \%$ in the US and 24.1 to 35\% in Europe. Characteristics associated with receipt of NAAT included clinical factors such as age, poor performance status, comorbidities, and uncontrolled concomitant conditions, as well as sociodemographic factors such as female sex, unmarried status, and lower income. Survival was diminished among patients receiving NAAT, with reported median overall survival values ranging from 1.2 to 4.8 months compared to 5 to 14.4 months with NIC. These findings suggest a proportion of patients who are candidates for NIC receive NAAT, potentially forfeiting the survival benefit of active antileukemic treatment.
\end{abstract}

Keywords Acute myeloid leukemia $\cdot$ Treatment patterns $\cdot$ Undertreatment $\cdot$ Non-intensive chemotherapy $\cdot$ Real-world evidence

\section{Introduction}

AML is a life-threatening hematologic malignancy most common in older adults (SEER 2020). In the US alone, 19,940 new cases of AML and 11,180 deaths were projected for 2020 (SEER 2020). The prognosis of AML is bleak, particularly for patients aged 65 years and older who represent $58.9 \%$ of new cases in the US but account for $72.5 \%$ of AML-related deaths. In this population, 1-year survival rates

Elizabeth Hubscher

elizabeth.hubscher@pshta.com

1 Purple Squirrel Economics, a Cytel Company, Waltham, MA, USA

2 Pfizer Inc., New York, NY, USA are $\leq 30 \%$ (Dores et al. 2012; Ocias et al. 2016; Osca-Gelis et al. 2015; Shah and Ghimire 2014; Thein et al. 2013).

The poor prognosis of older patients with AML is a function of a complex set of biological and clinical factors. The population is highly heterogenous, exhibiting wide variability in health history, performance and functional status, comorbidities, organ dysfunction, polypharmacy, cytogenetic abnormalities, and social support that may influence the ability to tolerate treatment, resistance to available therapies, and likelihood of receiving optimal treatment (Eleni et al. 2010; Wheatley et al. 2009). Other disease factors, such as etiology (de novo, secondary, or therapy-related AML [t-AML]) and baseline bone marrow blasts also may impact a patient's disease course, treatment, and outcomes (DiNardo et al. 2016; Stone et al. 2019).

Newly diagnosed patients with AML may be treated with IC, followed by hematopoietic stem cell transplant (HSCT) 
and/or consolidation therapy. Lower-intensity treatments such as hypomethylating agents (HMAs) have been available to patients who are not candidates for IC since the mid2000s. Newer non-intensive treatments such as glasdegib and venetoclax, as well as targeted treatments, including fms-like tyrosine kinase 3 (FLT3) and isocitrate dehydrogenase (IDH) inhibitors, and the antibody-drug conjugate gemtuzumab ozogamicin, became available after 2016 (Hitzler and Estey 2019; Lancet 2018; Wang 2014). All patients receive some form of BSC, which includes some combination of infection control, pain relief, transfusions, and antiemesis.

Determining candidacy for IC or NIC is not standardized. Existing international treatment guidelines recommend against using age as the only determining factor, but no consensus algorithm has been published to date (Döhner et al. 2017; Tallman et al. 2019). In addition to age, comorbidities, performance status (PS), and disease characteristics, physicians account for patient factors including treatment preference, financial and other resources, and available support systems (Kantarjian et al. 2010). Evidence to aid decisionmaking is often conflicting and limited, in part because clinical trials generally exclude patients on the basis of poor PS, confounding comorbidities, and advanced age (Kantarjian et al. 2010; Medeiros et al. 2018). Both providers and patients are tasked with balancing the risks of adverse events and treatment-related mortality and benefits of anti-cancer therapy (intensive or non-intensive) for older patients with AML (Wang 2014).

With a lack of consensus and complex considerations, the optimal treatment of older patients with AML presents a distinct challenge for physicians. Unsurprisingly, wide variation in treatment selection exists (Loberiza et al. 2014); moreover, analyses of real-world data regarding therapeutic utilization in AML suggest that a substantial number of patients receive NAAT despite published evidence that AML patients, including those over age 65 , benefit from receiving active antileukemia treatment (Menzin et al. 2002; Oran and Weisdorf 2012).

We sought to examine real-world treatment patterns in AML, including factors associated with the selection of NAAT as opposed to NIC or IC and survival outcomes to provide insight into the factors that influence treatment selection and the impact of undertreatment, or receipt of NAAT by a patient who may benefit from NIC treatment.

\section{Methods}

We conducted a focused literature review that concentrated on rates of undertreatment, as well as patient characteristics and survival outcomes, using an iterative hybrid of pearl growing and snowball search methods (LibGuides 2020;
Ramer 2005; Zwakman et al. 2018). Briefly, an initial broad search was conducted to identify a set of core publications. The abstracts of initial records were screened and core publications were selected based on the relevance of patient population, interventions, and outcomes to the research question as well as large sample sizes that were representative of the heterogenous AML patient population. These core publications were then used for prospective searches (e.g., cited by) and retrospective bibliographic searches, as well as the identification of new index terms for additional searches.

The initial search was conducted in PubMed and employed the following search terms: "treatment pattern", "treatment decision" and "treatment utilization" and the MeSH heading "Acute Myeloid Leukemia". Search limitations were defined as English language articles only, dated between January 2010 and the date of the initial search (May 2020).

\section{Results}

A total of 831 initial abstract records were identified, from which nine core publications were selected and used for additional prospective, retrospective, and index-term searches. Overall, 86 records that described the proportion of patients receiving either IC, NIC, or NAAT were identified. Among these, 25 reported the proportion of patients who received NAAT. Most of the identified studies were retrospective analyses of large databases, while some analyzed registries and multicenter data. Eleven studies analyzed single-center data or were prospective. Several regions were represented, including the US, EU, UK, Asia, Scandinavia, Israel, and Brazil. Forty-seven studies limited populations to patients $>60$ years or had a median age of $\geq 60$ years. Among 24 studies reporting median patient age, the pooled average was 70.6 years (range $36-78$ ).

\section{Rates of no active antileukemic treatment}

Among the 25 studies reporting the proportion of patients receiving NAAT, the majority (57\%) were retrospective observational studies. Ten were single-center, while the rest were multicenter or analyzed registries or large databases, such as Programa para el Tratamiento de Hemopatias Malignas (PETHEMA), California Cancer Registry, National Cancer Database (NCDB), Surveillance Epidemiology and End Results Program (SEER) database, Centers for Medicare and Medicaid Services' claims, or commercially available claims databases. The payer databases provided data for both inpatient and outpatient medication claims. One study was prospective (Berger et al. 2019). The number of included patients varied widely, from 61 in a single-center study to 98,293 in an analysis of the NCDB. Overall, the 
identified studies represented a total of 220,569 patients with AML from regions including the US, EU, UK, Japan, Brazil, India, Israel, and Serbia.

In the US, the percentage of patients who received NAAT ranged from 10 to $61.4 \%$, with a weighted average of $30 \%$, whereas $24.1-35 \%$ of patients in the EU received NAAT (Table 1). Rates of active treatment in the rest of the world varied widely. In India, $65.5 \%$ of patients received NAAT (Kanakasetty et al. 2019). According to a small single-center study by Neaman et al. in 2019, $15.6 \%$ of Israeli patients with AML received NAAT (Neaman 2020).

\section{Temporal trends}

In identified studies, rates of NAAT among patients with AML show a downward trend in the last two decades (Hagiwara et al. 2018; Medeiros et al. 2015; Meyers et al. 2013; Zeidan et al. 2019). According to an analysis of the SEERMedicare database, patients diagnosed in 2000 or later were more likely to receive antileukemia treatment than patients diagnosed between 1997 and 1999: the odds ratio (OR) was 1.139 for those diagnosed from 2000 to 2003 and 1.310 for those diagnosed from 2004 to 2007 (Meyers et al. 2013). This effect was durable in more recent years. In an analysis of the NCDB, patients diagnosed with AML after the reference period of 2003-2006 were more likely to receive antileukemia treatment; for those diagnosed from 2007 to 2010, the OR was 1.11 (95\% CI 1.08-1.15), and for those diagnosed from 2011 to 2013, the OR was 1.27 (95\% CI 1.22-1.31) (Goyal et al. 2015).

Four studies provided some annual rates of NAAT, which were scatter plotted, and trend lines (dashed lines) were added subsequently (Fig. 1).

\section{Factors associated with treatment selection}

\section{Patient characteristics}

Twelve studies analyzed the characteristics of patients in either the antileukemia treatment or NAAT groups. Patients who received NAAT tended to be older, with 12 studies reporting an association between increased age and decreased likelihood of receiving antileukemia treatment. The majority of studies reported patient comorbidity burden
Table 1 Reported rates of NAAT in identified studies

\begin{tabular}{|c|c|c|c|c|}
\hline Study reference & Country & NAAT (\%) & $\begin{array}{l}\text { Study popula- } \\
\text { tion }(N)\end{array}$ & Date range \\
\hline Talati (2020) & US & 28 & 980 & 1995-2016 \\
\hline Bhatt (2018) & US & 25.3 & 61,775 & 2003-2011 \\
\hline Мa (2016) & US & 43 & 1139 & 2005-2015 \\
\hline Medeiros (2015) & US & 60 & 8336 & 2000-2009 \\
\hline Meyers (2013) & US & 57 & 4058 & $1997-2007$ \\
\hline Tu (2019) & US & 18 & 2879 & 2012-2018 \\
\hline Willner (2019) & US & 26.2 & 61 & 2000-2017 \\
\hline Zeidan (2019) & US & 52.7 & 14,089 & 2001-2013 \\
\hline Oran (2012) & US & 61.4 & 5480 & 2000-2007 \\
\hline Percival (2019) & US & 10 & 442 & 2014-2016 \\
\hline Goyal (2015) & US & 24 & 98,293 & 2003-2013 \\
\hline Hagiwara (2018) & US & 32 & 9455 & 2007-2016 \\
\hline Solomon (2020) & US & 19 & 323 & 2009-2017 \\
\hline Berger (2019) & France & 30.2 & 592 & 2009-2014 \\
\hline Deschler (2013) & Germany & 24.1 & 195 & 2004-2008 \\
\hline Heiblig (2017) & France & 35 & 302 & 2000-2014 \\
\hline Acuna-Cruz (2020) & Spain & 28 & 2637 & 1999-2013 \\
\hline Martinez-Cuadrón (2020) & Spain and Portugal & 25 & 8521 & 1990-2019 \\
\hline Yanada (2015) & Japan & 27 & 158 & 2004-2012 \\
\hline Serna $(2020)^{*}$ & Spain & 29.6 & 135 & 2009-2019 \\
\hline Colovic (2012) & Serbia & 34.8 & 210 & 2001-2006 \\
\hline Sandes (2011) & Brazil & 51.6 & 31 & 2003-2008 \\
\hline Neaman (2020) & Israel & $50 ; 15.6$ & $44 ; 32$ & 2017-2018; 2019 \\
\hline Kanaksetty (2019) & India & 65.5 & 402 & 2013-2017 \\
\hline
\end{tabular}

$N$ number; $N A A T$ no active antileukemia treatment

*This study population included only patients who were not candidates for IC 
Fig. 1 Rates of NAAT over time

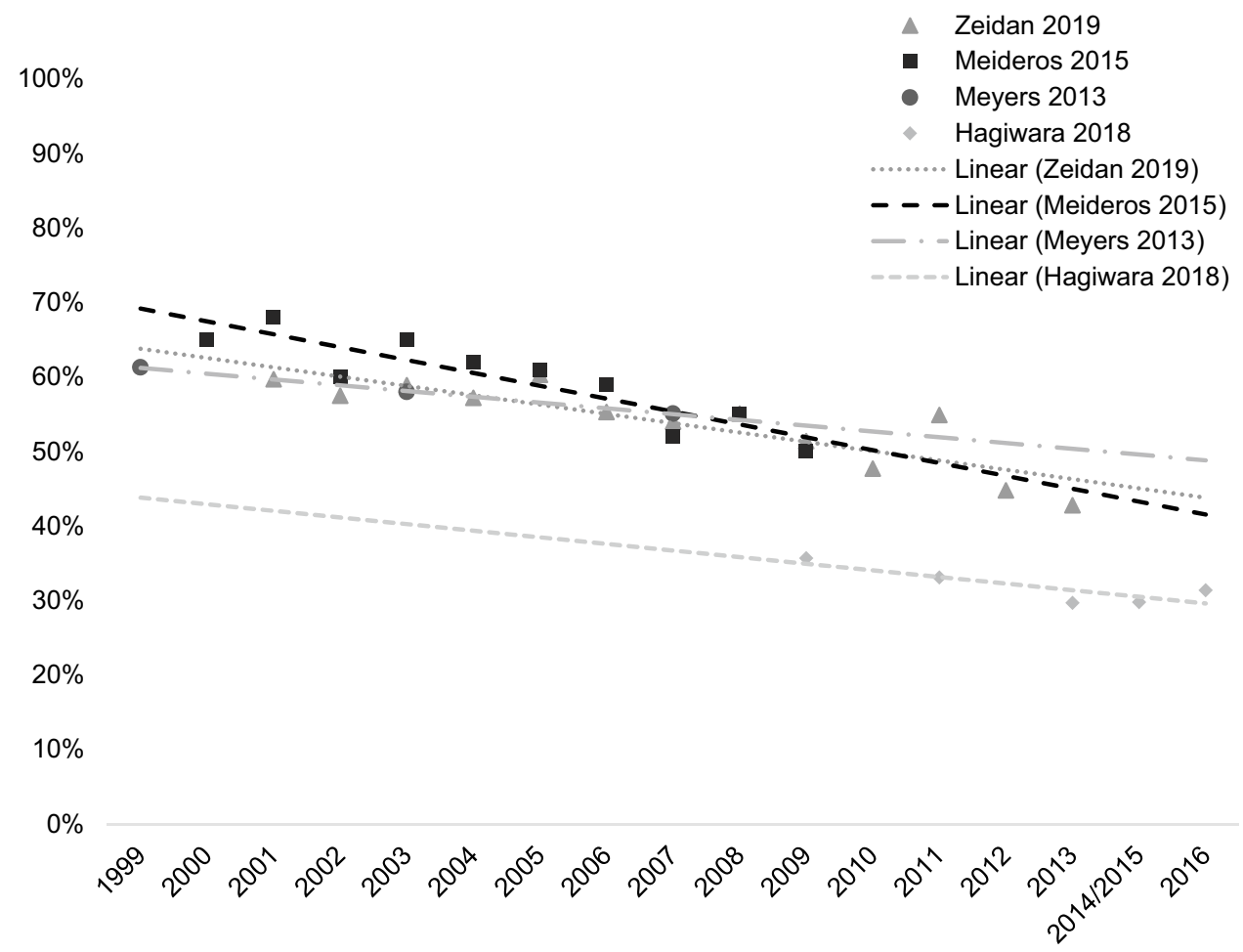

and/or PS. The Charlson Comorbidity Index was the most commonly used assessment (nine studies), followed by National Cancer Institute Comorbidity Index (three studies), HCT-CI (two studies), and Elixhauser comorbidity score (one study). Overall, a higher comorbidity index or presence of a chronic comorbid condition that was not optimally controlled was the second most common factor associated with receipt of NAAT: nine of 13 studies reported an association (Table 2). The most frequently reported comorbidities were cardiovascular disease and diabetes. Chronic kidney disease,

Table 2 Clinical characteristics associated with NAAT status in identified studies

\begin{tabular}{llll}
\hline Study reference & Age & Comorbidity burden & PS \\
\hline Oran (2012) & $\checkmark \geq 70$ years & $\checkmark$ CCI $\geq 1$ & - \\
Meyers (2013) & $\checkmark \geq 75$ years & $\checkmark$ CCI $\geq 1$ & - \\
Medeiros (2015) & $\checkmark$ NAAT patients were significantly older (81 vs 75 years, $p<0.0001)$ & $\checkmark$ CCI $\geq 1$ & $\checkmark$ PPI \\
Goyal (2015) & $\checkmark$ Elderly & $\checkmark \geq 1$ comorbid condition & - \\
Patel (2015) & $\checkmark$ Increasing age* & $\checkmark$ CCI $\geq 1$ & - \\
Yanada (2015) & $\checkmark \geq 75$ years & - & $\checkmark$ ECOG PS 3-4 \\
Hirsch (2015) & $\checkmark$ NAAT patients were significantly older (79 vs 77 years, $p=0.0082)$ & - \\
Heiblig (2017) & - & - & $\checkmark$ ECOG PS $\geq 2$ \\
Hagiwara (2018) & $\checkmark \geq 60$ years & $\checkmark$ Higher mean CCI; history & - \\
& & of COPD, CKD, dementia, & diabetes \\
Bhatt (2018) & $\checkmark \geq 60$ years & $\checkmark$ Higher \\
Zeidan (2019) & $\checkmark \geq 70$ years & $\checkmark \geq 3$ comorbidities & - \\
Martinez (2020) & $\checkmark$ Increasing age* & - \\
Acuna-Cruz & $\checkmark$ NAAT patients were significantly older (79 vs 76 years, $p<0.001)$ & - \\
(2020) & & & $\checkmark$ Worse ECOG PS \\
\hline
\end{tabular}

$\checkmark$ Associated with receipt of NAAT, followed by study definition of characteristic; - not assessed

$C C I$ charlson comorbidity index; $C K D$ chronic kidney disease; $C O P D$ chronic obstructive pulmonary disease; ECOG PS Eastern Cooperative Oncology Group performance status; NAAT no active antileukemia treatment; PPI poor performance indicators

*Study observed a significant difference in NAAT for all patient groups above youngest group (18-29 and 18-21 years); however, the effect was more marked in patients with increasing age and $>60$ years 
chronic obstructive pulmonary disease, and dementia were also associated with a higher probability of receiving NAAT compared to any active treatment (Hagiwara et al. 2018). Though few studies provided data regarding treatment decision criteria, the presence of an active suboptimally managed medical condition, severe cardiovascular disease, or diabetes and cardiovascular disease were provided as reasons for selecting NAAT (Colovic et al. 2012; Serna 2020). Either advanced age or the presence of a comorbidity was cited as the reason for choosing not to treat a patient with chemotherapy in $11.4 \%$ of cases in the NCDB (Medeiros et al. 2015).

With respect to PS, the Eastern Cooperative Oncology Group (ECOG) PS was the most frequently used (eight studies). In five studies, the proportion of patients with poor ECOG PS (ranging from $\geq 2$ to $3-4$ ) was higher among the group that received NAAT compared to those patients receiving chemotherapy or targeted treatment. Poor PS (ECOG PS 4) was a provided reason for the selection of NAAT in one study in Serbia (Colovic et al. 2012). Because the SEER database does not capture PS, Medeiros et al. assessed poor performance indicators (PPI) such as supplemental oxygen, wheelchair, home health, and skilled nursing claims. The presence of PPI in the year prior to AML diagnosis was significantly higher among the population receiving NAAT (Medeiros et al. 2015). A higher number of prior outpatient pharmacy claims was also observed in the patient population NAAT (Hagiwara et al. 2018).

\section{Disease characteristics}

Several of the identified studies characterized disease characteristics in both the antileukemia treatment and NAAT populations (Table 3). In two studies, rates of secondary AML were higher in the NAAT population (Oran and Weisdorf 2012; Medeiros et al. 2015). Patients with a prior history of myelodysplastic syndrome (MDS) or with t-AML were less likely to receive antileukemia treatment. History of previous MDS was a criteria for selecting NAAT among patients who did not have poor PS in two studies (Colovic et al. 2012; Sandes et al. 2011). Of note, an analysis of the PharMetrics Plus-IMS Hospital Charge Detail Master database demonstrated a positive association between the history of MDS and receipt of chemotherapy (Hagiwara et al. 2018). While the rate of NAAT was $32 \%$ in the total AML population, it was $28 \%$ among patients with t-AML or AML with myelodysplastic-related changes.

Patients with adverse cytogenetic risk were also more likely to receive NAAT in studies including patients with AML in the US, France, Spain, and Portugal (Bhatt et al. 2018; Hirsch et al. 2015; Martinez-Cuadron 2020). Unfavorable cytogenetics were reported criteria for receiving NAAT according to a treatment algorithm for elderly patients in an academic hospital in Brazil (Sandes et al.
Table 3 Disease-related characteristics associated with NAAT status

\begin{tabular}{llll}
\hline Study reference & Type of AML & $\begin{array}{l}\text { Cytogenetic risk } \\
\text { category }\end{array}$ & $\begin{array}{l}\text { Other } \\
\text { hema- } \\
\text { tologic } \\
\text { factor }\end{array}$ \\
\hline Oran (2012) & Secondary AML & - & - \\
Medeiros (2015) & Secondary AML & - & - \\
Hirsch (2015) & - & Unfavorable risk & - \\
Hagiwara (2018) & No* & - & - \\
Martinez (2020) & - & Adverse & $\begin{array}{l}\text { Higher } \\
\text { base- } \\
\end{array}$ \\
& & & line \\
& & & WBC \\
& & & and \\
& & & BMB \\
\hline
\end{tabular}

$A M L$ acute myeloid leukemia; $B M B$ bone marrow blast; $W B C$ white blood cell

*Secondary AML was positively associated with receipt of antileukemia treatment in this study

- not assessed

2011). Other factors reported in single studies were higher baseline bone marrow blasts and white blood cell counts (Martinez-Cuadron 2020).

\section{Sociodemographic factors}

In eight identified studies, sociodemographic factors were associated with a type of treatment (Table 4). Females were significantly less likely than males to receive antileukemia treatment in seven studies representing patients from the SEER-Medicare, NCDB, California Cancer Registry, and PharMetrics databases with index dates ranging from 1998 through 2016 (Hagiwara et al. 2018; Medeiros et al. 2015; Zeidan et al. 2019; Goyal et al. 2015; Bhatt et al. 2018; Patel et al. 2015). In an analysis of the SEER database from 1997 to 2007, females had an OR of receipt of antileukemia treatment of 0.944 compared to males; however, this was not statistically significant (Meyers et al. 2013).

The effect of race on treatment selection was unclear. In three studies including patients from the PharMetrics database, the California Cancer Registry, and the NCDB, Black race was associated with lower odds of receiving antileukemia treatment (OR range 0.67-0.85) (Meyers et al. 2013; Bhatt et al. 2018; Patel et al. 2015); however, this association was not observed in separate analyses of the SEERMedicare database covering patients diagnosed between 2000 and 2013 (Oran and Weisdorf 2012; Medeiros et al. 2015; Zeidan et al. 2019). One analysis of the NCDB from 2003 to 2013 revealed higher odds of systemic treatment for Black patients with AML compared to White patients (OR $1.20 ; 1.13-1.26)$ (Goyal et al. 2015). 
Table 4 Sociodemographic characteristics associated with NAAT in identified studies

\begin{tabular}{lllllll}
\hline Reference & Sex & Race/ethnicity & Marital status & Economic status & Education & Treatment setting \\
\hline Medeiros (2015) & Female & No & Unmarried & Lower income & - & - \\
Oran (2012) & Female & No & - & Lower income & - & - \\
Goyal (2015) & Female & No** & - & Lower income & - & Community hospital \\
Hagiwara (2018) & Female & - & - & - & - & - \\
Meyers (2013) & No & Black & - & - & - & - \\
Patel (2015) & Female & Black & - & - & Lower income, less insured & Lower educa- \\
Bhatt (2018) & Female & Black & - & & Non-academic \\
& & & & & tional status & center, lower \\
& & & Unmarried & Lowest income quartile & - & - \\
Zeidan (2019) & Female* & No & & &
\end{tabular}

*Except age 66-69, where men were more likely to receive NAAT

**Black patients had increased odds of receiving active treatment compared to White patients (OR $1.20 ; 1.13-1.26)$

${ }^{a}$ Income was defined based on median household income by zipcode and assessed in quartiles $(0-25$ th $\%, 26$ th- 50 th $\%$, 51st-75th\%, 76th100 th\%); actual numeric income ranges varied depending on year of data collection

- not assessed; No: association was not observed

Other identified factors associated with not receiving antileukemia treatment included lower household income status (five studies) and unmarried status (two studies). Patients who were treated at academic centers and/or hospitals with higher volume, travelled longer distances to receive treatment, reported a visit with an oncologist or hematologist within 1 year of AML diagnosis, or received the influenza vaccine were more likely to receive antileukemia treatment (Goyal et al. 2015; Bhatt et al. 2018). Patients with a diagnosed mental disorder or disability had an increased odds of receiving NAAT (OR 1.43 and 2.31, respectively) (Zeidan et al. 2019).

\section{Patient preference}

Though the majority of cases not receiving chemotherapy in the NCDB did not specify a reason, $11.3 \%$ cited patient or family refusal (Bhatt et al. 2018). In a study of patients in India between 2014 and $2017,53.3 \%$ of patients refused active treatment for a variety of reasons including insufficient or absent family support, travel/relocation constraints, and financial limitations (Kanakasetty et al. 2019). In a discrete choice experiment, two preference patterns emerged for either short-term side effect avoidance or complete remission achievement (Richardson et al. 2020). Women and patients over the age of 60 years were significantly more likely to be side-effect avoidant, whereas patients with private insurance were more likely to seek remission. Interestingly, a survey of patients and practitioners revealed a discordance between patients' recollection of discussions with healthcare providers regarding treatment options. Furthermore, $21 \%$ of surveyed patients reported discussing BSC alone and $28 \%$ reported that the treatment decision-making process did not match their preference (El-Jawahri et al. 2019).

\section{Outcomes with treatment}

Overall, patients who received NAAT in the identified studies had worse overall survival (OS) than those who received active antileukemia treatment, with reported median OS ranging from 1.2 to 4.8 months vs 5 to 14.4 months, respectively (Table 5). Across these heterogeneous patient populations, the weighted average OS among patients receiving NAAT was 1.7 months, whereas it was 8.0 months among patients receiving NIC. In two studies, patients who received treatment had 45-65\% reduction in risk of death compared with patients who received NAAT (Medeiros et al. 2015; Willner et al. 2019).

Table 5 Survival outcomes with NAAT and NIC

\begin{tabular}{llc}
\hline Study reference & $\begin{array}{l}\text { mOS with NAAT } \\
\text { (months) }\end{array}$ & $\begin{array}{l}\text { mOS } \\
\text { with NIC } \\
\text { (months) }\end{array}$ \\
\hline Talati (2020) & 2.1 & 14.4 \\
Ma (2016) & 4.8 & 8.6 \\
Medeiros (2015) & 1.5 & 5.0 \\
Willner (2019) & 2.0 & 10.0 \\
Martinez (2020) & 1.2 & 9.0 \\
Acuna-Cruz (2020) & 1.2 & 7.8 \\
Heiblig (2017) & 2.6 & 11.5 \\
\hline
\end{tabular}

mOS median overall survival; $N A A T$ no active antileukemia treatment; NIC non-intensive treatment 


\section{Discussion}

Despite an observable trend toward increased use of antileukemia treatment among patients with AML in recent years, there remains a substantial portion of patients who do not receive therapy beyond BSC. In our review, the most common factor associated with receipt of NAAT was increasing age. Current AML treatment guidelines recommend that age should not be the sole determinant of a patient's eligibility for treatment, however, the pervasiveness of this finding suggests physicians may continue to strongly consider age in treatment decisions. Survival gains for younger patients with AML in recent decades have not been observed to the same degree in older patients (SEER 2020; Thein et al. 2013; Shallis et al. 2019). This disparity may be due, at least in part, to undertreatment. Older age is inherently linked to other important characteristics that may influence a patient's ability to tolerate and benefit from treatment, including frailty, presence of comorbidities, decreased organ function, and diminished PS. Frailty is not universally measured and was not reported in the studies that we identified; however, other characteristics such as ECOG PS and comorbidities were analyzed in univariate analyses in a number of the studies, with mixed results. Of note, among studies reporting a relationship between older age and receipt of NAAT, poor PS or elevated comorbidity burden were also independently linked to receipt of NAAT. Reported ORs showed a more dramatic impact with age than comorbidity burden; however, the observed age effect may have been attenuated if adjustments for comorbidity burden had been performed (Oran and Weisdorf 2012; Meyers et al. 2013; Goyal et al. 2015; Patel et al. 2015). Bhatt et al. (2018) was the only identified study that reported results of multivariable modeling examining the interaction of age and comorbidity burden on the likelihood of receiving NAAT (Bhatt et al. 2018). Among patients over age 41 years, elevated risk of receiving NAAT with increasing age holds and was consistent across patient groups with increasing Charlson Comorbidity Index. Future analyses of multivariable models will help to further elucidate the role of age alone as well as the contribution of comorbidities and other important characteristics including frailty and PS on treatment decisions and patients' ability to tolerate and benefit from treatment.

It is possible that some physicians employ criteria intended to determine a patient's candidacy for IC to exclude them from NIC as well. According to a survey of 100 hematologists, $5 \%$ expressed a preference for using BSC only to treat patients who were either elderly or not candidates for IC (Portugal and Nucci 2019). Percival et al. (2019) reported the lowest rate of NAAT (10\%). This study was unique in that researchers examined patients aged 75 years or older who met AML clinical trial inclusion criteria of PS 0-2, glomerular filtration rate at or below $60 \mathrm{ml} / \mathrm{min}$, alanine transaminase at or below twice the upper limit of normal, bilirubin $\leq 1.5 \mathrm{mg} / \mathrm{dl}$, and measures of cardiac health including no history of myocardial infarction, congestive heart failure, or left ventricular ejection fraction below $49 \%$, suggesting that clinicians were more likely to treat patients that met IC treatment criteria (Percival et al. 2019). In identified studies, clinical factors such as poor PS and the presence of comorbidities were associated with NAAT. Two studies specified poor PS as a criterion for receiving NAAT (Colovic et al. 2012; Sandes et al. 2011). Concomitant conditions such as cardiovascular disease, diabetes, and chronic kidney disease were reported as decision drivers for NAAT (Colovic et al. 2012; Bhatt et al. 2018). While such biologic factors may confer a poorer prognosis, in some cases these patients benefit from treatment. In a clinical trial of glasdegib, nearly $67 \%$ of patients with ECOG PS 2 treated with glasdegib plus low-dose cytarabine achieved complete remission (Cortes et al. 2020). Additionally, trials of both glasdegib and venetoclax allowed patients with cardiovascular disease and post-hoc analyses of extended follow-up data demonstrated survival benefits over low-dose cytarabine alone (Cortes et al. 2020; Wei et al. 2020).

Our review also identified reports of other non-clinical factors that were more common among patients who received NAAT. Several US studies demonstrated an association between female sex, lower household income, and unmarried status and the receipt of NAAT (Oran and Weisdorf 2012; Hagiwara et al. 2018; Medeiros et al. 2015; Meyers et al. 2013; Zeidan et al. 2019; Goyal et al. 2015; Bhatt et al. 2018). Though none of the identified studies established a correlation between these patient characteristics and the reported treatment decisions, such patterns warrant further exploration. An association between Black race and receipt of NAAT was reported in three studies; however, this association was not observed in the SEER-Medicare database and in one of two analyses of the NCDB. Though evidence is mixed, sociodemographic factors do appear to influence survival outcomes. There is evidence that the risk of death among patients with AML is higher in those who live alone and are not married as well as among patients with lower income levels (Costa et al. 2016). Black Americans with AML also have worse survival than other races (Byrne et al. 2011; Pulte et al. 2013).

The current literature review identified studies comprising a large population of patients diagnosed with AML over nearly three decades, from 1990 to 2019. Analyzed data represented a wide range of regions and were derived from several different real-world evidence sources, including payer databases, registries, and single- and multicenter data. 
While the identified studies examined treatment patterns, characteristics of patients receiving types of therapy, and factors involved in decision making as well as overall patient outcomes, there was a great deal of variability in the data reporting, both in terms of analyses and the available input data depending on the source. In terms of our search strategy, though the pearl growing/snowball method is designed to provide comprehensive retrieval, the selection of core publications creates the possibility of selection bias, and ultimately the review is limited by the quality and quantity of retrieved studies and data. The majority of identified studies were retrospective observational analyses not designed to elucidate reasons for treatment selection. Moreover, it is impossible to discern whether worse outcomes among patients with NAAT are a result of undertreatment or if these patients were treated supportively because they had poor prognoses at the time of diagnosis. While non-clinical factors appear to play a role in driving treatment decisions, our assessment of the data is descriptive and did not ascertain the influence of certain factors in terms of treatment selection or outcomes. Longitudinal analyses of individual patient data would provide more robust and comprehensive insights.

Since 2017, new NIC options beyond low-dose cytarabine and HMAs have been available to extend survival in patients with AML who are not candidates for IC; however, the findings of this review suggest a substantial proportion of patients who might benefit from such treatment do not receive it (Wang 2014; Shallis et al. 2019; Bories et al. 2018; Griffiths et al. 2020; Palmieri et al. 2020). Across identified studies, patients treated with NIC experienced longer OS than those who received NAAT. Based on the proportion of patients in the identified studies who received NAAT instead, this may represent a potentially substantial amount of life years lost to undertreatment. It is important to note that most of the treatment and outcome data in this review were based on timeframes before these treatments were widely available; thus, practice shifts based on recent NIC approvals may not be reflected. Regardless, treatment decisions for patients with AML remain challenging and must consider the patient's overall situation. Certain non-clinical factors appear to negatively impact a patient's likelihood of receiving antileukemia treatment. Enhanced understanding of key considerations influencing treatment selection and survival outcomes will aid in the development of consensus guidelines to guide and inform complex clinical decisions. Furthermore, improved access and support for patients in more vulnerable populations will create better opportunities for parity and optimal care.

Author contribution EH and SS designed the study. EH conducted the literature search, collected data, performed data analysis, interpreted data, and wrote the manuscript. SS, TB, AB, VW, AR-S, and PD reviewed the manuscript, interpreted data, and provided expertise.

Funding Pfizer provided financial support to an author who is an employee of the vendor for the development of the manuscript.

Availability of data and material All data is provided in the manuscript.

Code availability Not applicable.

\section{Declarations}

Conflict of interest EH is an employee of Purple Squirrel Economics, who were paid consultants to Pfizer in connection with the development of this manuscript. SS, TB, AB, VW, AR-S, and PD are employees of and own stock in Pfizer, Inc.

Ethics approval Local ethics committee approval was not required because this study was based on published data.

Consent to participate Not applicable.

Consent for publication Not applicable.

Open Access This article is licensed under a Creative Commons Attribution 4.0 International License, which permits use, sharing, adaptation, distribution and reproduction in any medium or format, as long as you give appropriate credit to the original author(s) and the source, provide a link to the Creative Commons licence, and indicate if changes were made. The images or other third party material in this article are included in the article's Creative Commons licence, unless indicated otherwise in a credit line to the material. If material is not included in the article's Creative Commons licence and your intended use is not permitted by statutory regulation or exceeds the permitted use, you will need to obtain permission directly from the copyright holder. To view a copy of this licence, visit http://creativecommons.org/licenses/by/4.0/.

\section{References}

Acuna-Cruz E (2020) Evolving treatment patterns and outcomes of $3637 \geq 60$ year-old patients with newly diagnosed AML reported to the PETHEMA epidemiologic registry. EHA 25. https://libra ry.ehaweb.org/eha/2020/eha25th/294488. Accessed 16 June 2020

Berger E, Delpierre C, Despas F et al (2019) Are social inequalities in acute myeloid leukemia survival explained by differences in treatment utilization? Results from a French longitudinal observational study among older patients. BMC Cancer 19:883. https://doi.org/ 10.1186/s12885-019-6093-3

Bhatt VR, Shostrom V, Gundabolu K, Armitage JO (2018) Utilization of initial chemotherapy for newly diagnosed acute myeloid leukemia in the United States. Blood Adv 2:1277-1282. https:// doi.org/10.1182/bloodadvances.2018019125

Bories P, Lamy S, Simand C et al (2018) Physician uncertainty aversion impacts medical decision making for older patients with acute myeloid leukemia: results of a national survey. Haematologica 103:2040-2048. https://doi.org/10.3324/haematol.2018.192468

Byrne MM, Halman LJ, Koniaris LG et al (2011) Effects of poverty and race on outcomes in acute myeloid leukemia. Am J Clin Oncol 34:297-304. https://doi.org/10.1097/COC.0b013e3181dea934

Colovic M, Colovic N, Radojkovic M et al (2012) Induction chemotherapy versus palliative treatment for acute myeloid leukemia in 
a consecutive cohort of elderly patients. Ann Hematol 91:13631370. https://doi.org/10.1007/s00277-012-1478-2

Cortes JE, Heidel FH, Fiedler W et al (2020) Survival outcomes and clinical benefit in patients with acute myeloid leukemia treated with glasdegib and low-dose cytarabine according to response to therapy. J Hematol Oncol 13:1-12. https://doi.org/10.1186/ s13045-020-00929-8

Costa LJ, Brill IK, Brown EE (2016) Impact of marital status, insurance status, income, and race/ethnicity on the survival of younger patients diagnosed with multiple myeloma in the United States. Cancer 122:3183-3190. https://doi.org/10.1002/cncr.30183

Deschler B, Ihorst G, Platzbecker U et al (2013) Parameters detected by geriatric and quality of life assessment in 195 older patients with myelodysplastic syndromes and acute myeloid leukemia are highly predictive for outcome. Haematologica 98(2):208-216. https://doi.org/10.3324/haematol.2012.067892

DiNardo CD, Garcia-Manero G, Pierce S et al (2016) Interactions and relevance of blast percentage and treatment strategy among younger and older patients with acute myeloid leukemia (AML) and myelodysplastic syndrome (MDS). Am J Hematol 91:227232. https://doi.org/10.1002/ajh.24252

Döhner H, Estey E, Grimwade D et al (2017) Diagnosis and management of AML in adults: 2017 ELN recommendations from an international expert panel. Blood 129:424-447. https://doi.org/ 10.1182/blood-2016-08-733196

Dores GM, Devesa SS, Curtis RE et al (2012) Acute leukemia incidence and patient survival among children and adults in the United States, 2001-2007. Blood 119:34-43. https://doi.org/10. 1182/blood-2011-04-347872

Eleni LD, Nicholas ZC, Alexandros S (2010) Challenges in treating older patients with acute myeloid leukemia. J Oncol 2010:943823. https://doi.org/10.1155/2010/943823

El-Jawahri A, Nelson-Lowe M, VanDusen H et al (2019) Patient-clinician discordance in perceptions of treatment risks and benefits in older patients with acute myeloid leukemia. Oncologist 24:247254. https://doi.org/10.1634/theoncologist.2018-0317

Goyal G, Nawal L, Mosalpuria K, Silberstein PT (2015) Impact of socioeconomic, demographic and health system factors on therapy in acute myeloid leukemia. Blood 126:3316-3316. https://doi.org/ 10.1182/blood.V126.23.3316.3316

Griffiths EA, Carraway HE, Chandhok NS, Prebet T (2020) Advances in non-intensive chemotherapy treatment options for adults diagnosed with acute myeloid leukemia. Leuk Res 91:106339. https:// doi.org/10.1016/j.leukres.2020.106339

Hagiwara M, Sharma A, Chung KC, Delea TE (2018) Healthcare resource utilization and costs in patients with newly diagnosed acute myeloid leukemia. J Med Econ 21:1119-1130. https://doi. org/10.1080/13696998.2018.1513847

Hirsch P, Qassa G, Marzac C et al (2015) Acute myeloid leukemia in patients older than 75: prognostic impact of FLT3-ITD and NPM1 mutations. Leuk Lymphoma 56:147-150. https://doi.org/10.3109/ 10428194.2014.913288

Hitzler J, Estey E (2019) Gemtuzumab ozogamicin in acute myeloid leukemia: act 2, with perhaps more to come. Haematologica 104:7-9. https://doi.org/10.3324/haematol.2018.205948

Heiblig M, Le Jeune C, Elhamri M et al (2017) Treatment patterns and comparative effectiveness in elderly acute myeloid leukemia patients (age 70 years or older): the Lyon-university hospital experience. Leuk Lymphoma 58(1):110-117. https://doi.org/10.1080/ 10428194.2016.1180688

Kanakasetty GB, Chethan R, Lakshmaiah KC et al (2019) Treatment patterns and comparative analysis of non-intensive regimens in elderly acute myeloid leukemia patients-a real-world experience from India. Ann Hematol 98:881-888. https://doi.org/10.1007/ s00277-019-03600-6
Kantarjian H, Ravandi F, O’Brien S et al (2010) Intensive chemotherapy does not benefit most older patients (age 70 years or older) with acute myeloid leukemia. Blood 116:4422-4429. https://doi. org/10.1182/blood-2010-03-276485

Lancet JE (2018) Is the overall survival for older adults with AML finally improving? Best Pract Res Clin Haematol 31:387-390. https://doi.org/10.1016/j.beha.2018.09.005

Jonkers J LibGuides: Information Literacy History: Search methods. https://libguides.rug.nl/c.php?g=470628\&p=3218096. Accessed 21 Oct 2020

Loberiza FR, Cannon AC, Cannon AJ, Bierman PJ (2014) Insights on practice variations in the management of lymphoma and leukemia. Leuk Lymphoma 55:2449-2456. https://doi.org/10.3109/10428 194.2014.881480

Martinez-Cuadron D (2020) Characteristics and treatment strategies of secondary acute myeloid. EHA 25. https://library.ehaweb.org/ eha/2020/eha25th/294501. Accessed 16 June 2020

Ma E, Bonthapally V, Chawla A et al (2016) An evaluation of treatment patterns and outcomes in elderly patients newly diagnosed with acute myeloid leukemia: a retrospective analysis of electronic medical records from US community oncology practices. Clin Lymphoma Myeloma Leuk 16(11):625-636.e3. https://doi.org/ 10.1016/j.clml.2016.08.006

Medeiros BC, Satram-Hoang S, Hurst D et al (2015) Big data analysis of treatment patterns and outcomes among elderly acute myeloid leukemia patients in the United States. Ann Hematol 94:11271138. https://doi.org/10.1007/s00277-015-2351-x

Medeiros BC, Satram-Hoang S, Momin F, Parisi M (2018) Increase in chemotherapy use and associated survival benefit among medicare-aged patients with Acute myeloid leukemia (AML). Blood 132:3591-3591. https://doi.org/10.1182/blood-2018-99-111281

Menzin J, Lang K, Earle CC et al (2002) The outcomes and costs of acute myeloid leukemia among the elderly. Arch Intern Med 162:1597-1603. https://doi.org/10.1001/archinte.162.14.1597

Meyers J, Yu Y, Kaye JA, Davis KL (2013) Medicare fee-for-service enrollees with primary acute myeloid leukemia: an analysis of treatment patterns, survival, and healthcare resource utilization and costs. Appl Health Econ Health Policy 11:275-286. https:// doi.org/10.1007/s40258-013-0032-2

Neaman M (2020) Changes In The Approach To Care And In Treatment Outcomes In Elderly Acute Myeloid Leukemia Patients In The Era Of Venetoclax Based Regimens: A Single Center Experience. EHA 25. https://library.ehaweb.org/eha/2020/eha25th/ 294490. Accessed 11 June 2020

Ocias LF, Larsen TS, Vestergaard H et al (2016) Trends in hematological cancer in the elderly in Denmark, 1980-2012. Acta Oncol Stockh Swed 55(Suppl 1):98-107. https://doi.org/10.3109/02841 86X.2015.1115124

Oran B, Weisdorf DJ (2012) Survival for older patients with acute myeloid leukemia: a population-based study. Haematologica 97:1916-1924. https://doi.org/10.3324/haematol.2012.066100

Osca-Gelis G, Puig-Vives M, Saez M et al (2015) Is survival in myeloid malignancies really improving? A retrospective 15 -year population-based study. Leuk Lymphoma 56:896-902. https://doi.org/ 10.3109/10428194.2014.947610

Palmieri R, Paterno G, De Bellis E et al (2020) Therapeutic choice in older patients with acute myeloid leukemia: a matter of fitness. Cancers. https://doi.org/10.3390/cancers12010120

Patel MI, Ma Y, Mitchell B, Rhoads KF (2015) How do differences in treatment impact racial and ethnic disparities in acute myeloid leukemia? Cancer Epidemiol Prev Biomark 24:344-349. https:// doi.org/10.1158/1055-9965.EPI-14-0963

Percival M-EM, Othus M, Mirahsani S et al (2019) Frequency, and effect on survival, of ineligibility for clinical trials in newly diagnosed acute myeloid leukemia and high-grade myeloid 
neoplasms. Blood 134:3824-3824. https://doi.org/10.1182/ blood-2019-130834

Portugal RD, Nucci MLM (2019) Current treatment preferences in acute myeloid leukemia: a survey in Brazil. Hematol Transfus Cell Ther. https://doi.org/10.1016/j.htct.2019.07.005

Pulte D, Redaniel MT, Jansen L et al (2013) Recent trends in survival of adult patients with acute leukemia: overall improvements, but persistent and partly increasing disparity in survival of patients from minority groups. Haematologica 98:222-229. https://doi. org/10.3324/haematol.2012.063602

Ramer SL (2005) Site-ation pearl growing: methods and librarianship history and theory. J Med Libr Assoc 93:397-400

Richardson DR, Crossnohere NL, Seo J et al (2020) Age at diagnosis and patient preferences for treatment outcomes in AML: a discrete choice experiment to explore meaningful benefits. Cancer Epidemiol Biomark Prev 29:942-948. https://doi.org/10.1158/ 1055-9965.EPI-19-1277

Sandes AF, da Costa Ribeiro JC, Barroso RS et al (2011) Improving the outcomes of elderly patients with acute myeloid leukemia in a Brazilian University Hospital. Clin Sao Paulo Braz 66:1335-1340. https://doi.org/10.1590/s1807-59322011000800005

SEER (2020) SEER*Explorer Application-AML. https://seer.cancer. gov/explorer/application.html?site $=96 \&$ data_type $=4 \&$ graph type $=2 \&$ compareBy $=$ survival_interval $\&$ chk_survival_inter val_1 $=1 \&$ sex $=1 \&$ race $=1 \&$ age_range $=157 \&$ hdn_stage $=101 \&$ advopt_precision $=1 \&$ advopt_display $=2$. Accessed 8 May 2020

Serna A (2020) Is less more in the treatment of elderly patients with acute myeloid leukemia? The impact of the introduction of clinical trials at our institution. EHA 25. https://library.ehaweb.org/eha/ 2020/eha25th/294553. Accessed 11 June 2020

Solomon SR, Solh M, Jackson KC et al (2020) Real-world outcomes of unselected elderly acute myeloid leukemia patients referred to a leukemia/hematopoietic cell transplant program. Bone Marrow Transplant 55(1):189-198. https://doi.org/10.1038/ s41409-019-0675-1

Shah BK, Ghimire KB (2014) Improved survival among older acute myeloid leukemia patients-a population-based study. Acta Oncol Stockh Swed 53:935-938. https://doi.org/10.3109/0284186X. 2014.889851

Shallis RM, Wang R, Davidoff A et al (2019) Epidemiology of acute myeloid leukemia: recent progress and enduring challenges. Blood Rev 36:70-87. https://doi.org/10.1016/j.blre.2019.04.005

Stone A, Zukerman T, Flaishon L et al (2019) Efficacy outcomes in the treatment of older or medically unfit patients with acute myeloid leukaemia: a systematic review and meta-analysis. Leuk Res 82:36-42. https://doi.org/10.1016/j.leukres.2019.05.007

Talati C, Dhulipala VC, Extermann MT et al (2020) Comparisons of commonly used front-line regimens on survival outcomes in patients aged 70 years and older with acute myeloid leukemia.
Haematologica 105(2):398-406. https://doi.org/10.3324/haema tol.2018.208637

Tallman MS, Wang ES, Altman JK et al (2019) Acute myeloid leukemia, version 3.2019, NCCN clinical practice guidelines in oncology. J Natl Compr Canc Netw 17:721-749. https://doi.org/10. 6004/jnccn.2019.0028

Thein MS, Ershler WB, Jemal A et al (2013) Outcome of older patients with acute myeloid leukemia: an analysis of SEER data over 3 decades. Cancer 119:2720-2727. https://doi.org/10.1002/cncr. 28129

Wang ES (2014) Treating acute myeloid leukemia in older adults. Hematol Am Soc Hematol Educ Program 2014:14-20. https:// doi.org/10.1182/asheducation-2014.1.14

Tu N, Islam Z, Fliss AE, Salas M (2019) An evaluation of major comorbidities and treatment patterns of newly diagnosed acute myeloid leukemia patients: a retrospective analysis of electronic medical records from US. Blood. 134(Supplement_1):5106-5106. https://doi.org/10.1182/blood-2019-124517

Wei AH, Montesinos P, Ivanov V et al (2020) Venetoclax plus LDAC for newly diagnosed AML ineligible for intensive chemotherapy: a phase 3 randomized placebo-controlled trial. Blood 135:21372145. https://doi.org/10.1182/blood.2020004856

Wheatley K, Brookes CL, Howman AJ et al (2009) Prognostic factor analysis of the survival of elderly patients with AML in the MRC AML11 and LRF AML14 trials. Br J Haematol 145:598-605. https://doi.org/10.1111/j.1365-2141.2009.07663.x

Willner CA, Chisti MM, Soriano M, Huang J (2019) Analyzing how elderly acute myeloid leukemia patients are treated, an institutional experience. Blood 134:5849-5849. https://doi.org/10.1182/ blood-2019-121560

Yanada M, Okamoto A, Inaguma Y et al (2015) The fate of patients with acute myeloid leukemia not undergoing induction chemotherapy. Int J Hematol. 102(1):35-40. https://doi.org/10.1007/ s12185-015-1786-0

Zeidan AM, Podoltsev NA, Wang X et al (2019) Temporal patterns and predictors of receiving no active treatment among older patients with acute myeloid leukemia in the United States: a populationlevel analysis. Cancer 125:4241-4251. https://doi.org/10.1002/ cncr.32439

Zwakman M, Verberne LM, Kars MC et al (2018) Introducing PALETTE: an iterative method for conducting a literature search for a review in palliative care. BMC Palliat Care 17:82. https://doi. org/10.1186/s12904-018-0335-Z

Publisher's Note Springer Nature remains neutral with regard to jurisdictional claims in published maps and institutional affiliations. 